\title{
Geriatric Assessment in Oncology
}

\author{
Nitish Garg ${ }^{1}$ Rushabh Kothari ${ }^{2}$ \\ ${ }^{1}$ Medical Oncology Resident, Vydehi Institute of Medical Sciences \& \\ Research Center, Bangalore, Karnataka, India \\ 2 Department of Medical Oncology, canCURE Cancer Center, Narayana \\ Multispeciality Hospital, Ahmedabad, Gujarat, India
}

Ind J Med Paediatr Oncol 2021;42:457-460.
Address for correspondence Nitish Garg, MD, DNB, DM, Consultant Medical Oncologist, Sri Shankara Cancer Hospital, Basavangudi, Bangalore. 560004, Karnataka, India (e-mail: nitish_jeenu@yahoo.co.in).
Today, in practice of an oncologist almost $54 \%$ new cases and $70 \%$ of the patients who die due of cancer are above 65years. ${ }^{1}$ Thus, geriatric oncology is a very important aspect for all the oncologist. Lung cancer, prostate cancer, breast cancer, and many other common cancers have median population in geriatric age. Elderly patients are different from young and young adults in many significant ways; these include physiological changes, increased prevalence of cancers, varied presentation of common diseases, and variable responses to treatment and the interactions of the drugs due to multiple comorbidities. The elderly patients are usually not included in the clinical trials due to their comorbidities, and hence, these patients require comprehensive assessment before starting the treatment. There are few guidelines that address the evaluation process and treatment in elderly patients.

Oncologists face issues for assessing the patients, whom to be chosen for treatment and for whom to provide best supportive care. The definition of elderly patients differs among various study groups starting from 60 to 70 years. The chronological age of a person can help assessing the physiological status but does not still define it completely about the tolerance to the cancer treatment. There exists wide heterogenicity among the patients of the same age to any aggressive therapy to cancer.

The elderly patient has lesser chances to get the standard treatment as provided to the younger adults due to their comorbidities, varied toxicities due to physiological status, and access to health-care facilities. ${ }^{2-8}$ The aging process decreases the physiological reserve of multiple organs and increases the chances of frailty and increased chances of toxicity even without comorbidities, manifesting as weight loss, fatigue, and decreased activity. Hence, it is important to identify the frail patients and recognize the potential benefits and adverse effects of treatment.
Geriatric Assessment: It is a multidisciplinary process involving the functional, psychological, nutritional, and medical assessment of an elderly patient before starting the cancer treatment, and it helps in finding the problems not found by routine evaluation. ${ }^{9}$

Initially, it was developed for the assessment of general geriatric population for detecting aging-related issues slowly rolled over to oncology clinics. The initial approaches in clinics included assessment of functional, psychological and mental, nutritional and cognitive, and economic status of the patient, but these were time consuming. Then came the assessment by a questionnaire form by Hurria ${ }^{10}$ and now recently shifted to electronic models of assessment with various algorithms. As it is very time consuming, hence came the role of screening assessment for patients who needs comprehensive geriatric assessment (CGA). ${ }^{10}$ The major and important domains of CGA are as follows:

1. Demography and social status

2. Comorbidities

3. Functional status

4. Cognition

5. Polypharmacy

6. Nutrition

7. Geriatric syndromes

How do a complete geriatric assessment help us?

1. Predicts response to treatment and toxicities-several tools like chemotherapy risk assessment scale for high age patients (CRASH) and cancer and aging research group (CARG) score use geriatric assessment data for predicting toxicities of chemotherapy.
DOI https://doi.org/ 10.1055/s-0041-1735596. ISSN 0971-5851.
(C) 2021. Indian Society of Medical and Paediatric Oncology. All rights reserved.

This is an open access article published by Thieme under the terms of the Creative Commons Attribution-NonDerivative-NonCommercial-License, permitting copying and reproduction so long as the original work is given appropriate credit. Contents may not be used for commercial purposes, or adapted, remixed, transformed or built upon. (https://creativecommons.org/ licenses/by-nc-nd/4.0/)

Thieme Medical and Scientific Publishers Pvt. Ltd., A-12, 2nd Floor, Sector 2, Noida-201301 UP, India 
2. Predicts patient's vulnerability-helps in identifying abnormalities in almost $50 \%$ of population that are not found by routine evaluation. Most common among them are poor functional status (40.1\%), poor nutrition (37.6\%), depression (27.2\%), and cognitive impairment (19\%).

3. Helps in treatment decisions.

4. Helps on survival.

5. Helps to assess functional decline due to treatment.

6. Better assessment of dosages of drugs in view of comorbidity status.

7. Helps in planning interventions for the management of specific issues involving nutrition, social support, economic support, and physical therapy. ${ }^{10}$

Optimum cutoff age for patients for whom genetic assessment has to be done is not recommended clearly, but guidelines recommend that all elderly patients have to undergo CGA. The problem with CGA is that it is very time and resource consuming; hence, certain screening tools are used to decide who to go for CGA. The screening tools available are as follows:

1. Geriatric 8-best sensitivity and highly studied.

2. Senior Adult Oncology Program-2.

3. Abbreviated CGA includes geriatric depression assessment and Mini-Mental State Examination (MMSE).

4. Vulnerable Elderly Survery-13.

5. Fried Frailty Criteria.

6. Triage Risk Screening Tool (TRST).

None of these tools are better or preferred over the other. The specificity for abnormal CGA is highest for abbreviated CGA (97\%); maximum sensitivity is given by SAOP-2 or TRST criteria. The patients who are positive on screening test should be taken up for CGA. This can be completed by patient or caregiver within 20 minutes and the electronic tools with algorithm provide details of interventions necessary for improvement in patient condition. Even if the CGA cannot be performed, the screening tools provide useful information required for the management of cancer in elderly.

Other ways of assessment include:

1. Time Up and Go (TUG): it provides assessment of patient's mobility and balance and ability to avoid falls. TUG $>13$ seconds is associated with increased risk of falls and poor balance. It is done using a chair, stopwatch, and 3-minute walkway.

2. Geriatric Depression Scale-15 (GDS-15): It helps predicting the functional decline of the patient on cancer treatment. A score of $>5$ in a self-assessment 15 -point tool is considered abnormal.

For predicting the toxicities among patients, following tools are used:

1. Chemotherapy risk assessment scale for high age patients (CRASH)

2. Cancer and aging research group (CARG)

These tools are validated by various prospective studies and they recommend either of them can be used in clinical practice.
Collection of suitable data for assessment is the most important tool for GA. This data for patients can be collected either by:

1. Mailed CGA-It was used at Durham for male oncology patients to evaluate their pain, quality of life, functional status, financial and spiritual well-being, social support, and psychological status.

2. Self-administered CGA-It was developed by CALGB and Cancer in Older Adult committee. Most of them are selfevaluation by the patient, but three domains-functional, cognition, and performance status-are evaluated by the clinician.

3. Electronic CGA-Memorial Sloan Kettering Cancer Center (MSKCC) developed electronic rapid fitness assessment for preoperative evaluation.

4. Clinical assessment-This method involves assessment with the help of Multidimensional Assessment Protocol for Cancer, which includes an evaluation of the socioeconomic status, cognitive status, depression, physical performance, disability, and tumor characteristics. Time taken is 27 minutes.

Assessment of frailty and other domains of GA is discussed in the following text. ${ }^{11}$

\section{Frailty}

Frailty is one of the biggest factors to be assessed before starting treatment and the physiological age has bigger role to play than chronological age. Patients who are old with no comorbidities but with good functional status are considered as Fit older adults and are treated with same treatment as young adults, but this does not hold true for frail patient as they are more vulnerable to toxicities from chemotherapy if same dose is given.

Frailty is defined as a syndrome of advancing age that is characterized by chronic inflammation, sarcopenia, immune dysregulation, increased apoptosis, and loss of resilience, leading to loss of functional reserve of most organs. Several surrogate markers are used to recognize the frailty syndrome, like elevated cytokines and chemokines-interleukin ${ }^{6}$, reduced insulin growth factor-1, low levels of DHEAS and Leptin and changes in white blood cell counts. These changes affects mitochondrial DNA and genetic material of the individual, which leads to decreased functional reserve, dysregulation of various physiological systems and not achieving physiological homeostasis post stressor.

It is evaluated by frailty phenotype-weight loss, exhaustion, low physical activity, loss of grip, and slow gait speed, or by frailty index (FI) developed by Canadian Study of Health and Aging, which is 70-item based tool based on concept of accumulation of deficits. FI was considered cumbersome to calculate, hence based on Frality index, a, clinical frailty score was developed and has been used. (-Table $\mathbf{1}$ ).

Cancer and frailty are closely related and frailty in cancer patient increases the chances of mortality and morbidity. Also, cancer can lead to increased frailty or it may be the consequence of therapy in cancer survivors. Frailty and aging 
Table 1 Clinical frailty scale

\begin{tabular}{|l|l|}
\hline Scale & Fitness of frailty \\
\hline Category 1 & Very fit: robust, active, energetic, motivated; the fittest group for their age \\
\hline Category 2 & Well: no active disease symptoms, but less fit than people in category 1 \\
\hline Category 3 & Managing well: disease symptoms are well controlled compared with those in category 4 \\
\hline Category 4 & Vulnerable: not dependent on others but symptoms limit activities; they are "slowed up" \\
\hline Category 5 & Mildy frail: limited dependence on others for some instrumental activities of daily living \\
\hline Category 6 & Moderately frail: help is needed with both instrumental and basic activities of daily living \\
\hline Category 7 & Severely frail: completely dependent on others for self-care or terminally ill \\
\hline
\end{tabular}

can help understanding the disease biology, and detecting frailty may help in the identification of patients on high risk of mortality but still alone frailty may not be helpful as other factors contribute too; hence, it requires CGA.

\section{Comorbidity Assessment}

Comorbidities play an important role in pretreatment assessment of any patient and more in elderly. More the comorbidities, high the chances of treatment interruptions and complications during treatment. Pretreatment assessment is done by Charlson Co-morbidity Index or Cumulative Illness Rating Scale-Geriatrics.

\section{Cognitive Assessment}

As the age increases, chances of dementia increase and it doubles up after every 5 years after 65 years, and it increases further to $37 \%$ at the age of 85 years. Dementia with cancer is associated with delayed diagnosis, poor understanding with treatment, and poor compliance to treatment. The tools used for cognitive assessment include MMSE, Montreal Cognitive Assessment, and blessed orientation memory cognition test. As the patients are involved in shared decision making for the therapy it is unclear whether to obtain consent from an elderly patient with impaired cognition or not.

\section{Polypharmacy}

Polypharmacy is defined as the use of more than five drugs per day. ${ }^{12}$ Due to multiple comorbidities, patients may be on various medication that can alter the pharmacodynamics and pharmacokinetics of the cancer drugs leading to higher toxicities. Among the newly diagnosed cancer, polypharmacy was seen almost in $80 \%$ of the patients.

\section{Social Issues and Quality of Life}

Cancer diagnosis is associated with changes in social life that affects the quality of life of the patient at any age. Studies have shown social isolation and poor social support are associated with higher mortalities and increased toxicities of cancer treatment.

\section{Physical Function Assessment}

Many available scales are used for the assessment of physical function-Eastern Cooperative Oncology Group performance status, Karnofsky performance status, TUG, the Tinetti Gait and Balance scale and easier ways are - gait speed and grip strength. The Short Physical performance battery uses gait speed for evaluating strength and balance of lower extremity. Most importantly the patient has to be assessed for history of falls along with the other physical assessment. An elderly patient with higher incidence of falls or poor balance is associated with higher chemotherapy-related toxicities.

\section{Functional Assessment}

It includes the assessment of patient for activities of daily living whether they require any assistance in performing the activity. Activity of daily living (ADL) refers to basic self-care skills required to live at home independently and instrumental ADLs are those activities required to live independently in a community. The performance status of a patient does not provide adequate information regarding this and has to be asked by the clinician.

\section{Nutritional Assessment}

The incidence of malnutrition in elderly patient is very high and even higher for elderly cancer patients among them. Malnutrition and weight loss are important poor risk factors associated with cancer patients. Nutritional assessment should be a part of CGA and interventions to be provided to improve nutrition and prevent toxicities and treatment mortality. It can be evaluated with body mass index, skin fold thickness, mini-nutritional assessment score. MNA score has been validated among various studies and has shown that poor nutritional status correlates with higher treatment related mortality.

\section{Psychological Assessment}

Psychological stress and depression are the most common problems that impact the quality of life and cause functional decline in cancer patient and higher burden to caregivers. The prevalence of depression among various GA studies ranges from 10 to $65 \%$ and is associated with poor survival than those without depression. Patients should be assessed based on symptoms and objectively by GDS-15 or distress thermometer.

Over the past 10 years, GA has become part of geriatric oncology that helps to identify certain conditions, which can be missed by routine evaluation. It has not only larger impact on treatment decisions but also on individualizing the treatment for elderly at risk. International Society of Geriatric Oncology. SIOG recommends to incorporate CGA in all 
oncology treatment decisions. The choice of how detailed or comprehensive the assessment should be shall depend on the type of use and certain abbreviated tools can be still be used.

\section{Conflict of Interest}

None.

\section{References}

1 Surveillance, Epidemiology, and End Results (SEER) Cancer Statistics Review, 1975-2015. http://seer.cancer.gov/archive/csr/ 1975_2015/. Accessed July 05, 2021

2 Christman K, Muss HB, Case LD, Stanley V. Chemotherapy of metastatic breast cancer in the elderly. The Piedmont Oncology Association experience [see comment]. [see comment]JAMA 1992;268(01):57-62

3 Hurria A, Leung D, Trainor K, Borgen P, Norton L, Hudis C. Factors influencing treatment patterns of breast cancer patients age 75 and older. Crit Rev Oncol Hematol 2003;46(02):121-126

4 Mandelblatt JS, Hadley J, Kerner JF, et al. Patterns of breast carcinoma treatment in older women: patient preference and clinical and physical influences. Cancer 2000;89(03):561-573
5 Newcomb PA, Carbone PP. Cancer treatment and age: patient perspectives. J Natl Cancer Inst 1993;85(19):1580-1584

6 Merchant TE, McCormick B, Yahalom J, Borgen P. The influence of older age on breast cancer treatment decisions and outcome. Int J Radiat Oncol Biol Phys 1996;34(03):565-570

7 Bergman L, Dekker G, van Leeuwen FE, Huisman SJ, van Dam FS, van Dongen JA. The effect of age on treatment choice and survival in elderly breast cancer patients. Cancer 1991;67(09): 2227-2234

8 Bergman L, Kluck HM, van Leeuwen FE, et al. The influence of age on treatment choice and survival of elderly breast cancer patients in south-eastern Netherlands: a population-based study. Eur J Cancer 1992;28A(8-9):1475-1480

9 Loh KP, Soto-Perez-de-Celis E, Hsu T, et al. What every oncologist should know about geriatric assessment for older patients with cancer: young International Society of Geriatric Oncology position paper. J Oncol Pract 2018;14(02):85-94

10 Cohen HJ. Evolution of geriatric assessment in oncology. J Oncol Pract 2018;14(02):95-96

11 Korc-Grodzicki B, Holmes HM, Shahrokni A. Geriatric assessment for oncologists. Cancer Biol Med 2015;12(04):261-274

12 Golchin N, Frank SH, Vince A, Isham L, Meropol SB. Polypharmacy in the elderly. J Res Pharm Pract 2015;4(02):85-88 